\title{
5 Results Type of Impact
}

\subsection{Emission to Air}

\subsubsection{Greenhouse Gases}

Greenhouse gases are considered to have a major influence on climate warming. The Kyoto Protocol (United Nations Framework Convention on Climate Change, 1992) calls for reductions in the following greenhouse gases:

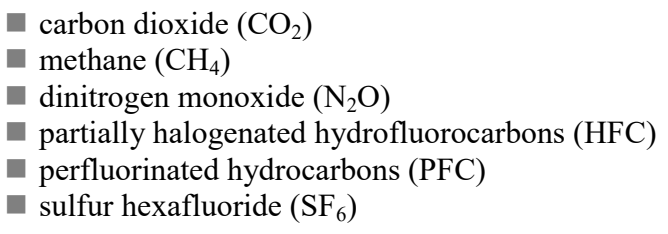

The Montreal Protocol on Substances that Deplete the Ozone Layer governs the reduction in CFC emissions and these substances, despite having a greenhouse effect, are thus not included in the Kyoto Protocol.

The Kyoto Protocol greenhouse gases (excluding CFCs) are abbreviated "GHG" below.

\subsubsection{Characterization}

The current publication of the "Intergovernmental Panel on Climate Change (IPCC)" (Forster, et al., 2007) serves as the reference for the global warming potential (GWP) of various gases. The reference substance is carbon dioxide $\left(\mathrm{CO}_{2}\right)$. The potentials are adapted as required to new scientific findings and new substances are described. The values vary depending on the time period over which the effects are summed. GWP100 values (100-year integration time) are normally used (see Table 1) and are also used for characterization in the present report.

The European Environment Agency statement (EEA 2014) is used here. The European Commission objective is to achieve a reduction of 80-95\% compared to 1990 emission levels by 2050 (European Commission EC 2011). In the present case a reduction in an amount of the minimum target value of $80 \%$ has been assumed in the first step, since it is at this level that the target worded in this way would first be achieved. If good progress is made, this objective may be further adjusted on subsequent revision of the data (cf. Ahbe et al. 2014). 
Table 1: Global warming potential for various greenhouse gases ${ }^{1}$

\begin{tabular}{|l|l|}
\hline Substance & Global warming potential (GWP) in CO2eq \\
\hline $\mathrm{CO}_{2}$ & 1 \\
$\mathrm{CH}_{4}$ & 25 \\
$\mathrm{~N}_{2} \mathrm{O}$ & 298 \\
$\mathrm{HFC}$ & $124-14,800$ \\
$\mathrm{PFC}$ & $7,390-17,700$ \\
$\mathrm{SF}_{6}$ & 22,800 \\
\hline
\end{tabular}

Table 2: Greenhouse gas emissions, EU-28

\begin{tabular}{|l|ll|}
\hline Reference year & $\begin{array}{l}\text { EU-28 GHG emissions (excluding CFCs) } \\
\text { in (million t } \mathrm{CO}_{2} \text { eq)/y }\end{array}$ & Source \\
\hline Baseline: 1990 & 5,626 & EEA 2014 \\
Current: 2012 & 4,544 & EEA 2014 \\
\hline Target: $2050(80 \%)$ & 1,125 & EC 2011 \\
\hline
\end{tabular}

Applying the calculation specification, the following eco-factor is thus obtained, here shown purely by way of example:

$$
E F_{\text {CO2eq }}=1 \cdot \frac{E P}{4,544,000 * \frac{10^{9} g}{a}} \cdot\left(\frac{4,544,000 * \frac{10^{9} g}{a}}{1,125,000 * \frac{10^{9} g}{a}}\right)^{2} \cdot \frac{10^{12}}{a}=0.00359 \frac{E P}{g}
$$

Table 3: EU-28 eco-factor for greenhouse gas emissions

\begin{tabular}{|l|l|}
\hline Reference year & Eco-factor in EP, EU-28/g $\mathrm{CO}_{2} \mathrm{eq}$ \\
\hline 2050 & 0.00359 \\
\hline
\end{tabular}

\subsubsection{Preliminary Remarks on Air Pollutants}

There are essentially two competing perspectives on this impact category: on the one hand, the non-binding targets according to "Thematic Strategy on Air Pollution" ((TSAP), European Commission 2005) and, on the other, the more recent, binding, but less demanding reduction targets according to the Gothenburg Protocol (ECE/

1 (Forster, et al., 2007), p. 212 
EB.AIR/114, 2012). In a written communication dated 10.7.2014, the EEA stated, in response to an enquiry, that the TSAP targets from 2005 should be considered outdated and that it was obvious that these targets could not be achieved within the originally planned periods. For this reason there should be a reorientation, which is described as follows:

"The European Commission completed a review of the EU air legislation and TSAP at the end of 2013. It has proposed new legal EU targets for 2020 (based on the Gothenburg ceilings) and also for 2030 (representing a further step of moving towards the EU objective of ensuring "levels of air quality that do not give rise to significant negative impacts on, and risks to human health and the environment" (EEA 2014a).

The new, binding definition based on the Gothenburg Protocol is thus used below, since this definition has also been agreed by the EU with the individual countries with regard to implementation. The actual values used in these calculations are those from 2010 , since they reflect the latest determined and established state. Since previous and future reduction efforts will in many cases result in lower annual loads, the eco-factor calculations will have to be adapted from time to time to take account of the actual, more recent emission loads.

\subsubsection{NMVOC}

NMVOC (non-methane volatile organic compounds):

In addition to their sometimes toxic effects, these are precursor substances for photooxidants and are harmful to human and plant health. According to the 31 st Federal Pollution Control Ordinance (article 2, paragraph 11), a volatile organic compound is defined as "an organic compound which, at 293.15 kelvin, has a vapour impact of 0.01 kilopascals or more or, under its particular conditions of use, has a corresponding volatility. The creosote fraction which, at 293.15 kelvin, exceeds this vapour impact is considered to be a volatile organic compound." Methane is not subsumed in this category.

The intention is, by 2020 , to reduce emissions by $28 \%$ in comparison with 2005 levels (ECE/EB.AIR/114, 2012).

Table 4: $\quad$ NMVOC emissions, EU-28

\begin{tabular}{|l|ll|}
\hline Reference year & EU-28 NMVOC emissions in (kt VOC)/y & Source \\
Baseline: 2005 & 8,842 & ECE/EB.AIR/114, 2012 \\
Actual load: 2010 & 7,500 & EEA (2013) \\
\hline Target: $2020(28 \%)$ & 6,366 & ECE/EB.AIR/114, 2012 \\
\hline
\end{tabular}


Applying the calculation specification, the following eco-factor is thus obtained:

Table 5: EU-28 eco-factor for NMVOC emissions

\begin{tabular}{|l|l|}
\hline Reference year & Eco-factor in EP, EU-28/g NMVOC \\
\hline 2020 & 0.1851 \\
\hline
\end{tabular}

\subsubsection{NO}

$\mathrm{NO}_{\mathrm{x}}$ (measured as $\mathrm{NO}_{2}$ ) contributes not only to ozone formation potential (POCP) but also to eutrophication and acidification. The intention is, by 2020 , to reduce emissions by $42 \%$ in comparison with 2005 levels (ECE/EB.AIR/114, 2012).

Table 6: $\quad \mathrm{NO}_{\mathrm{x}}$ emissions, EU-28

\begin{tabular}{|l|ll|}
\hline Reference year & EU-28 $\mathrm{NO}_{x}$ emissions in $(\mathrm{kt} \mathrm{NO}) / \mathrm{y}$ & Source \\
Baseline: 2005 & 11,355 & ECE/EB.AIR/114, 2012 \\
Actual load: 2010 & 9,000 & EEA (2013) \\
\hline Target: $2020(42 \%)$ & 6,585 & ECE/EB.AIR/114, 2012 \\
\hline
\end{tabular}

Applying the calculation specification, the following eco-factor is thus obtained:

Table 7: EU-28 eco-factor for $\mathrm{NO}_{\mathrm{x}}$ emissions

\begin{tabular}{|l|l|}
\hline Reference year & Eco-factor in EP, EU-28/g NO ${ }_{x}$ \\
\hline 2020 & 0.2076 \\
\hline
\end{tabular}

\subsubsection{SO}

$\mathrm{SO}_{\mathrm{x}}$ (measured as $\mathrm{SO}_{2}$ ) are harmful to the respiratory organs and are a precursor substance for acidic precipitation which in turn damages plants and buildings. The intention is, by 2020 , to reduce emissions by $59 \%$ in comparison with 2005 levels (ECE/ EB.AIR/114, 2012).

Table 8: $\quad \mathrm{SO}_{2}$ emissions, EU-28

\begin{tabular}{|l|ll|}
\hline Reference year & EU-28 $\mathrm{SO}_{2}$ emissions in $\left(\mathrm{kt} \mathrm{SO}_{2}\right) / \mathrm{y}$ & Source \\
Baseline: 2005 & 7,828 & ECE/EB.AIR/114, 2012 \\
Actual load: 2010 & 5,000 & EEA (2013) \\
\hline Target: $2020(59 \%)$ & 3,209 & ECE/EB.AIR/114, 2012 \\
\hline
\end{tabular}


Applying the calculation specification, the following eco-factor is thus obtained:

Table 9: EU-28 eco-factor for $\mathrm{SO}_{2}$ emissions

\begin{tabular}{|l|l|}
\hline Reference year & Eco-factor in EP, EU-28/g SO 2 \\
\hline 2020 & 0.4855 \\
\hline
\end{tabular}

\subsubsection{PM2.5}

Fine particulate matter consists of particles of very different origins: abrasion, geological and biological material, soot etc. Its effects range from health impairment via respiratory tract conditions to carcinogenicity. One distinguishing feature is maximum diameter, which is conventionally broken down into PM10 (particulate matter, max. 10 micrometres), PM2.5 and PM0.1 According to a communication from the German UBA (UBA, 2013c), in the absence of further information, a PM2.5 value can be estimated for approximation purposes by multiplying the PM10 value by a factor of 0.7 .

The intention is, by 2020 , to reduce emissions by $22 \%$ in comparison with 2005 levels (ECE/EB.AIR/114, 2012).

Table 10: PM2.5 emissions, EU-28

\begin{tabular}{|l|ll|}
\hline Reference year & EU-28 PM2.5 emissions in (kt PM2.5)/y & Source \\
Baseline: 2005 & 1,504 & ECE/EB.AIR/114, 2012 \\
Actual load: 2010 & 1,350 & EEA (2013) \\
\hline Target: $2020(22 \%)$ & 1,173 & ECE/EB.AIR/114, 2012 \\
\hline
\end{tabular}

Applying the calculation specification, the following eco-factor is thus obtained:

Table 11: EU-28 eco-factor for PM2.5 emissions

\begin{tabular}{|l|l|}
\hline Reference year & Eco-factor in EP, EU-28/g PM2.5 \\
\hline 2020 & 0.9812 \\
\hline
\end{tabular}

\subsection{8 $\mathrm{NH}_{3}$}

Ammonia $\left(\mathrm{NH}_{3}\right)$ is predominantly formed in agriculture, but also in industrial processes. $\mathrm{NH}_{3}$ plays a major part in forming acidic precipitation and is harmful to the ecosystem not only by acidification and overfertilisation but also by the formation of secondary pollutants. 
The intention is, by 2020 , to reduce emissions by $6 \%$ in comparison with 2005 levels (ECE/EB.AIR/114, 2012).

Table 12: $\mathrm{NH}_{3}$ emissions, EU-28

\begin{tabular}{|l|ll|}
\hline Reference year & EU-28 $\mathrm{NH}_{3}$ emissions in $\left(\mathrm{kt} \mathrm{NH}_{3}\right) / \mathrm{y}$ & Source \\
Baseline: 2005 & 3,813 & ECE/EB.AIR/114, 2012 \\
Actual load: 2010 & 3,500 & EEA (2013) \\
\hline Target: $2020(6 \%)$ & 3,584 & ECE/EB.AIR/114, 2012 \\
\hline
\end{tabular}

Applying the calculation specification, the following eco-factor is thus obtained:

Table 13: EU-28 eco-factor for $\mathrm{NH}_{3}$ emissions

\begin{tabular}{|l|l|}
\hline Reference year & Eco-factor in EP, EU-28/g NH 3 \\
\hline 2020 & 0.2725 \\
\hline
\end{tabular}

\subsection{Emissions Surface Water}

At EU-28 level, various recording systems have been used over an extended period. The EEA recommends using the current system, the "European Pollutant Release and Transfer Register" (E-PRTR) (EEA 2014b). This register replaced the preceding system EPER ${ }^{2}$ in 2009 and was designed to provide the public and relevant bodies with improved access to environmental data. The EEA simultaneously pointed out, however, that this register does not record all emission sources. The emphasis here is on relatively large industrial facilities that exceed a certain specified annual load threshold as "point sources". The E-PRTR system consequently does not include "diffuse sources", which also include those point dischargers which are below the reporting threshold. The EEA has carried out further investigations to identify the influence of "diffuse sources" and published the findings as a report (Deltares 2013).

The Deltares report investigated the proportion of diffuse sources in various discharge routes above and beyond the data already reported and recorded by the E-PRTR database (emissions from large point sources). Due a lack of data, the Deltares report explicitly provides no information about diffuse industrial emissions (i.e. those below the E-PRTR recording threshold), which nevertheless play a significant role in water pollution.

2 European Pollutant Emission Register 
In order to make good this shortcoming, German conditions were used as the basis for the following extrapolation: a factor corresponding to the ratio of the German values for emissions to surface water coordinated with the UBA to the German data from the Deltares report and the E-PRTR database was calculated for each emission (each water pollutant). These factors were then multiplied with those from the Deltares report and the E-PRTR database values. The proportion of diffuse industrial emissions derived from the better coordinated German data was thus applied in an identical ratio by extrapolation to EU-28. Extrapolation is thus carried out for each pollutant using the following scheme:

Table 14: Extrapolation scheme for pollutant discharge to surface water, EU-28

\begin{tabular}{|c|c|}
\hline Proportion from & Calculation \\
\hline E-PRTR (2012) & $\begin{array}{l}\text { Proportion of large point sources above } \\
\text { threshold value }\end{array}$ \\
\hline Deltares (2013) & $\begin{array}{l}+ \text { Proportion of diffuse sources and smaller } \\
\text { point sources excluding industrial sources }\end{array}$ \\
\hline $\begin{array}{l}\text { Extrapolation factor, determined from German } \\
\text { data }\end{array}$ & $\begin{array}{l}+ \text { Proportion of diffuse and smaller point } \\
\text { sources from industry }\end{array}$ \\
\hline Actual total, EU-26 (excl. HR \& MT) & Total EU-26 excluding Croatia and Malta \\
\hline Extrapolation factor for Croatia and Malta* & 1.009 \\
\hline Total actual load EU-28 & Total EU-28 (incl. HR \& MT) \\
\hline $\begin{array}{l}\text { Reduction target for this pollutant, determined } \\
\text { from German data }\end{array}$ & Reduction ratio* $=\mathrm{Fk} / \mathrm{F}$ (from $\mathrm{D}$ ) \\
\hline Total target load (Fk) & Actual load EU-28*(Fk/F) \\
\hline
\end{tabular}

*) pro rata to population (Eurostat 2014)

Target values: in response to a question, the EEA stated in a written communication of 2.10.2014 that no reduction targets for emissions to surface water have yet been defined as annual loads at EU-28 level (EEA 2014d). Various more local reduction programmes are being carried out, for example by the states bordering the North Atlantic or the Baltic Sea. The target values were thus selected in such a way that the ratios of actual and target values per impact correspond to those of the respective German data coordinated with the Federal Environment Agency.

The E-PRTR and Deltares study tables of values do not include any data for Croatia (HR) and Malta (MT). The data from the EU-26 format have therefore been extrapolated pro rata by population to EU-28 level.

This approach involves considerable inaccuracy in relation to these two individual countries. It is, however, better in the present case to make use of such an extra- 
polation than simply to treat the unknown quantities as non-existent and omit them. The investigations and discussions with the EEA have revealed that there is indeed still a need for further research and clarification in relation to water pollution. This need primarily relates firstly to recording current pollutant loads as completely as possible and secondly to defining objectives in order to specify desirable conditions for the future. The current state of the environment including all major pollutant loads, and the desired state of the environment, which can then be appropriately used for defining targets and as a guidance benchmark for industry, both have to be defined as clearly as possible to provide a basis for assessing industrial environmental impacts. Carrying out further research in this direction would have gone beyond the scope and time horizon of the present paper. The values were therefore extrapolated as described, subject to the described limitations.

Note regarding extrapolation by population size: Where country data are unavailable, a range of criteria may be used for extrapolating environmental impacts: population size, GDP, per capita GDP, energy generation or waste treatment parameters and many others, taken alone or in combination. Since plant technology and environmental regulations also vary considerably, it is largely impossible to state a single, generally applicable extrapolation formula. For reasons of simplicity, the ratio of populations was accordingly selected as the extrapolation criterion for the present paper.

\subsubsection{Nitrogen (as $N$ )}

Surface waters have variable sensitivity towards nitrogen compounds and two different effects are of significance: acidification and eutrophication.

Lakes which are naturally nitrogen-limited either year-round or temporarily are severely affected by nitrogen inputs. One major problem that extends beyond national borders is elevated nitrogen inputs to the oceans and their associated eutrophication. Nitrogen primarily enters the oceans via rivers, but also via the atmosphere, and, once in the oceans, it is the growth-limiting and hence the decisive factor for nutrient effects (UBA, 2009), (UBA, 2011). The sources provide the following data:

Table 15: Nitrogen emissions to surface water, EU-28

\begin{tabular}{|l|ll|}
\hline Proportion from & EU-28: N emissions (kt N)/y & Source \\
E-PRTR (2012) & 384.8 & EEA (2014a) \\
Deltares (2013) & 3,388 & Deltares (2013) \\
Extrapolation factor = 1.68 & 2,557 & Ahbe et al. (2014) \\
Actual total, EU-26 (excl. HR \& MT) & 6,330 & - \\
Actual total, EU-28 (incl. HR \& MT) & 6,387 & - \\
\hline Target (reduction ratio D =0.913) & 5,831 & Ahbe et al. (2014) \\
\hline
\end{tabular}


Applying the calculation specification, the following eco-factor is thus obtained:

Table 16: EU-28 eco-factor for nitrogen emissions

\begin{tabular}{|l|l|}
\hline Reference year & Eco-factor in EP, EU-28/g N \\
\hline 2014 & 0.1879 \\
\hline
\end{tabular}

\subsubsection{Phosphorus (as P)}

Elevated nutrient inputs (nitrogen and phosphorus inputs) into rivers, lakes, coastal waters and oceans generally result in eutrophication of these bodies of water (cf. 5.2.1 Nitrogen). The growth-limiting nutrient is usually phosphorus (UBA, 2009), (UBA, 2011).

The sources provide the following data:

Table 17: Phosphorus emissions to surface water, EU-28

\begin{tabular}{|l|ll|}
\hline Proportion from & EU-28: P emissions (kt P)/y & Source \\
E-PRTR (2012) & 40.2 & EEA (2014a) \\
Deltares (2013) & 131.9 & Deltares (2013) \\
Extrapolation factor = 1.89 & 153.17 & Ahbe et al. (2014) \\
Actual total, EU-26 (excl. HR \& MT) & 325.3 & - \\
Actual total, EU-28 (incl. HR \& MT) & 327.4 & - \\
\hline Target (reduction ratio D = 0.397) & 130.1 & Ahbe et al. (2014) \\
\hline
\end{tabular}

Applying the calculation specification, the following eco-factor is thus obtained:

Table 18: EU-28 eco-factor for phosphorus emissions

\begin{tabular}{|l|l|}
\hline Reference year & Eco-factor in EP, EU-28/g P \\
\hline 2014 & 19.34 \\
\hline
\end{tabular}

\subsubsection{Nickel}

"Heavy metals, being chemical elements, are not degradable in the environment. They only become a hazard for humans and the environment at elevated concentrations and if they can be absorbed by living organisms. Environmental concentrations are usually so low that no acute toxic effects occur. Long-term, chronic toxic effects, on the other hand, are to be anticipated if individual heavy metals are able to enter the food chain 
and accumulate in living organisms." (Hesse State Agency for the Environment and Geology (DE), 2013).

"When making an ecological assessment, the focus should primarily be on the phytotoxicity of the heavy metals copper, chromium, nickel and zinc, but on the human or animal toxicity of cadmium and lead." (State Agency for the Environment, Nature Conservation and Geology (DE), 2002).

Nickel is moreover one of the 33 priority substances under EU law (Directive 2008/ $105 / \mathrm{EC}$ of the European Parliament and of the Council on environmental quality standards in the field of water policy, amending Directive 2000/60/EC).

The sources provide the following data:

Table 19: Nickel emissions to surface water, EU-28

\begin{tabular}{|l|ll|}
\hline Proportion from & EU-28: Ni emissions (t Ni)/y & Source \\
E-PRTR (2012) & 263.0 & EEA (2014a) \\
Deltares (2013) & 472.9 & Deltares (2013) \\
Extrapolation factor = 4.68 & 2,708 & Ahbe et al. (2014) \\
Actual total, EU-26 (excl. HR \& MT) & 3,444 & - \\
Actual total, EU-28 (incl. HR \& MT) & 3,472 & - \\
\hline Target (reduction ratio D =0.472) & 1,638 & Ahbe et al. (2014) \\
\hline
\end{tabular}

Applying the calculation specification, the following eco-factor is thus obtained:

Table 20: EU-28 eco-factor for nickel emissions

\begin{tabular}{|l|l|}
\hline Reference year & Eco-factor in UBP, EU-28/g Ni \\
\hline 2014 & 1,293 \\
\hline
\end{tabular}

\subsubsection{Zinc}

Zinc discharges to surface water contribute to harming plant growth. The sources provide the following data: 
Table 21: Zinc emissions to surface water, EU-28

\begin{tabular}{|l|ll|}
\hline Proportion from & EU-28: Zn emissions (t Zn)/y & Source \\
E-PRTR (2012) & 1,796 & EEA (2014a) \\
Deltares (2013) & 2,619 & Deltares (2013) \\
Extrapolation factor = 4.38 & 14,923 & Ahbe et al. (2014) \\
Actual total, EU-26 (excl. HR \& MT) & 19,338 & - \\
Actual total, EU-28 (incl. HR \& MT) & 19,506 & - \\
\hline Target (reduction ratio D = 0.64) & 12,491 & Ahbe et al. (2014) \\
\hline
\end{tabular}

Applying the calculation specification, the following eco-factor is thus obtained:

Table 22: EU-28 eco-factor for zinc emissions

\begin{tabular}{|l|l|}
\hline Reference year & Eco-factor in EP, EU-28/g Zn \\
\hline 2014 & 125.0 \\
\hline
\end{tabular}

\subsubsection{COD}

DIN 38 409, part 41 (Deutsches Institut für Normung e.V., 1980) defines chemical oxygen demand (COD) as follows:

"The chemical oxygen demand (COD) of a volume of water is taken to be the volumerelated mass of oxygen which is equivalent to the mass of potassium dichromate which, under the working conditions of the method, reacts with the oxidisable substances present in the water." COD indicates the quantity of oxygen which is required to oxidise the organic compounds present in the water and is thus a measure of the concentration of organic substances in water.

All organic substances cause water pollution by oxygen consumption and thus limit the habitat for the fauna. Many substances may additionally have specific toxic effects which would have to be separately recorded (Frischknecht, Steiner, \& Jungbluth, 2009).

The sources provide the following data: 
Table 23: COD emissions to surface water, EU-28

\begin{tabular}{|l|ll|}
\hline Proportion from & EU-28: COD emissions (kt COD)/y & Source \\
E-PRTR (2012) & 1,669 & EEA (2014a) \\
Deltares (2013) & 1,800 & Deltares (2013) \\
Extrapolation factor = 1.18 & 624.4 & Ahbe et al. (2014) \\
Actual total, EU-26 (excl. HR \& MT) & 4,093 & - \\
Actual total, EU-28 (incl. HR \& MT) & 4,128 & - \\
\hline Target (reduction ratio D = 0.54) & 2,226 & Ahbe et al. (2014) \\
\hline
\end{tabular}

Applying the calculation specification, the following eco-factor is thus obtained:

Table 24: EU-28 eco-factor for COD emissions

\begin{tabular}{|l|l|}
\hline Reference year & Eco-factor in EP, EU-28/g COD \\
\hline 2014 & 0.833 \\
\hline
\end{tabular}

\subsubsection{Lead}

Humans, animals and plants can be harmed by emissions of lead. Lead is capable of accumulating in the food chain and as a result becoming more concentrated in the organism consuming it. The sources provide the following data:

Table 25: Lead emissions to surface water, EU-28

\begin{tabular}{|l|ll|}
\hline Proportion from & EU-28: Pb emissions (t Pb)/y & Source \\
E-PRTR (2012) & 175.0 & EEA (2014a) \\
Deltares (2013) & 452.2 & Deltares (2013) \\
Extrapolation factor = 3.9 & 1,819 & Ahbe et al. (2014) \\
Actual total, EU-26 (excl. HR \& MT) & 2,446 & - \\
Actual total, EU-28 (incl. HR \& MT) & 2,469 & - \\
\hline Target (reduction ratio D = 0.25) & 617.1 & Ahbe et al. (2014) \\
\hline
\end{tabular}

Applying the calculation specification, the following eco-factor is thus obtained: 
Table 26: EU-28 eco-factor for lead emissions

\begin{tabular}{|l|l|}
\hline Reference year & Eco-factor in EP, EU-28/g Pb \\
\hline 2014 & 6,483 \\
\hline
\end{tabular}

\subsubsection{Cadmium}

Cadmium has a toxic effect in humans and animals and can bioaccumulate, disrupting metabolic processes and possibly being carcinogenic. The sources provide the following data:

Table 27: Cadmium emissions to surface water, EU-28

\begin{tabular}{|l|ll|}
\hline Proportion from & EU-28: Cd emissions (t Cd)/y & Source \\
E-PRTR (2012) & $26.2^{*}$ & EEA (2014a) \\
Deltares (2013) & $52.56^{*}$ & Deltares (2013) \\
Extrapolation factor = 1.0 & - & Ahbe et al. (2014) \\
Actual total, EU-26 (excl. HR \& MT) & 78.76 & - \\
Actual total, EU-28 (incl. HR \& MT) & 79.55 & - \\
\hline Target (reduction ratio D = 0.25) & 19.91 & Ahbe et al. (2014) \\
\hline
\end{tabular}

*) numbers rounded

Applying the calculation specification, the following eco-factor is thus obtained:

Table 28: EU-28 eco-factor for cadmium emissions:

\begin{tabular}{|l|l|}
\hline Reference year & Eco-factor in EP, EU-28/g Cd \\
\hline 2014 & 200,705 \\
\hline
\end{tabular}

\subsubsection{Copper}

Even relatively low concentrations of copper emissions can have a disruptive and harmful effect on aquatic systems.

The sources provide the following data: 
Table 29: Copper emissions to surface water, EU-28

\begin{tabular}{|l|ll|}
\hline Proportion from & EU-28: Cu emissions (t Cu)/y & Source \\
E-PRTR (2012) & 418.0 & EEA (2014a) \\
Deltares (2013) & 642.02 & Deltares (2013) \\
Extrapolation factor = 3.12 & 2,252 & Ahbe et al. (2014) \\
Actual total, EU-26 (excl. HR \& MT) & 3,312 & - \\
Actual total, EU-28 (incl. HR \& MT) & 3,341 & - \\
\hline Target (reduction ratio D = 0.765) & 2,556 & Ahbe et al. (2014) \\
\hline
\end{tabular}

Applying the calculation specification, the following eco-factor is thus obtained:

Table 30: EU-28 eco-factor for copper emissions

\begin{tabular}{|l|l|}
\hline Reference year & Eco-factor in EP, EU-28/g Cu \\
\hline 2014 & 511.2 \\
\hline
\end{tabular}

\subsubsection{EPA-PAH16}

$\mathrm{PAH}$ are usually formed during the combustion of hydrocarbons and have a toxic and sometimes carcinogenic effect. PAH are various forms of fused benzene rings and several hundred of these compounds are known. Depending on purpose, various cumulative parameters, such as in this case EPA-PAH16, may be used for the substantially occurring compounds.

There are no statistical surveys of EPA-PAH16 emissions at EU-28 level, nor have any targets been set which can be used in this context. Omitting this environmental impact for this reason would considerably magnify the relative error in the assessments. Therefore, by way of a provisional solution, German conditions have been extrapolated pro rata to population to European conditions. It should be noted that, here too, this can at best be an approximation, constituting a first step in establishing the ESM assessment method with European data. The data must, however, be stated more accurately when the collected data and stated targets are updated in the future. 
Table 31: EPA-PAH16 emissions to surface water, EU-28

\begin{tabular}{|l|lll|}
\hline & EPA-PAH16 in t/y & Population & Source \\
Germany, actual load 2005 & 19.16 & Ahbe et al. (2014) \\
Germany, target load & 4.41 & Ahbe et al. (2014) \\
Population, EU-28 & & 503 million & Eurostat, population 2010 \\
Population, DE & & 81.8 million & Eurostat, population 2010 \\
Extrapolation, actual load EU-28 & 117.82 & & \\
\hline Extrapolation, target load EU-28 & 27.14 & & \\
\hline
\end{tabular}

Applying the calculation specification, the following eco-factor is thus obtained:

Table 32: EU-28 eco-factor for EPA-PAH16 emissions

\begin{tabular}{|l|l|}
\hline Reference year & Eco-factor in EP, EU-28/g EPA-PAH16 \\
\hline 2014 & 160,099 \\
\hline
\end{tabular}

\subsection{Consumption of Resources}

\subsubsection{Freshwater Consumption}

Freshwater consumption is defined (OECD, 2013) as any extraction of freshwater for production or consumption processes. Water used for power generation in hydroelectric power stations is not included in this definition.

The EEA provides information at relatively long intervals about absolute water extraction volumes by EU countries and about the countries' water exploitation index (WEI), which characterises the ratio of extraction volume to water supply, i.e. to the volume available to a country over the long term. The present paper adopts the OECD criterion necessary for defining "critical consumption" (Fk) and defines a WEI of $20 \%$ as the limit of tolerability ((OECD 2013), (Ahbe et al. 2014)). 
Table 33: OECD definition of water scarcity

\begin{tabular}{|l|ll|}
\hline OECD definition of water scarcity & WEI & Source \\
Moderate & $10-20 \%$ & OECD (2008) \\
Medium & $20-40 \%$ & OECD (2008) \\
High & over $40 \%$ & OECD (2008) \\
Critical extraction criterion (moderate to & $20 \%$ & OECD (2013) \\
medium) & & \\
\hline
\end{tabular}

The following values are obtained for the corresponding volumes:

Table 34: Water scarcity, EU-28

\begin{tabular}{|l|ll|}
\hline Water scarcity, EU-28 & Billion $\mathrm{m}^{3} / \mathrm{y}$ & Source \\
EU-28 water extraction & 270.8 & EEA 2012, 2010 \\
EU-28 water supply & 3,119 & EEA 2012 \\
Critical extraction volume, EU-28 & 623.8 & EEA 2012, 2010 \\
\hline
\end{tabular}

Applying the calculation specification, the following eco-factor is thus obtained:

Table 35: EU-28 eco-factor for freshwater consumption

\begin{tabular}{|l|l|}
\hline Reference year & Eco-factor in EP, EU-28/ $\mathrm{m}^{3}$ \\
\hline 2010 & 0.6959 \\
\hline
\end{tabular}

\subsubsection{Primary and Renewable Energy Consumption}

The assumption in ESM assessments is that primary energy consumption should be reduced, while the proportion of "renewable energy consumption" in primary energy consumption should increase. This means that, from the standpoint of the environmental impact assessment user, two Eco Factors must be stated with regard to energy scarcity which take account firstly of the described targets and secondly of the fact that the scarcity situation of the resource primary energy becomes less severe as renewable energies gradually replace the consumption of non-renewable primary energy.

An eco-factor is therefore stated both for "non-renewable primary energy consumption" and for "renewable energy consumption" (cf. also Ahbe et al. 2014).

The corresponding actual energy consumption values are obtained from stated Eurostat values for primary energy and renewable energies. 
Table 36: Actual energy consumption, EU-28

\begin{tabular}{|l|ll|}
\hline Actual energy consumption in EU-28 for 2012 & $\mathrm{PJ} / \mathrm{y}$ & Source \\
Actual primary energy consumption & 70,460 & Eurostat 2014a \\
Actual renewable energy consumption & 7,429 & Eurostat 2014b \\
Actual non-renewable primary energy consumption & 63,031 & Line 1 minus line 2 \\
\hline
\end{tabular}

Directive 2012/27/EU states a target value for total primary energy consumption in 2020. Directive 2009/28/EC sets country-specific targets for the proportion of final energy consumption to be accounted for by renewable sources. These proportions were applied to the calculated target values for total primary energy consumption for 2020 and multiplied by the ratio of EU-28 primary energy consumption to final energy consumption in order to obtain the desired value for the proportion of primary energy consumption accounted for by renewable sources in 2020 .

This calculation thus initially assumes that the ratio of primary energy to final energy will remain constant until 2020. Strictly speaking, this is not the case because the difference between the two types of energy largely consists of power station losses, the minimisation of which is currently being vigorously addressed. The timescale for upgrading power station technologies to distinctly better efficiency levels is, however, rather long. The change in the ratio of primary to final energy consumption which is to be anticipated by 2020 is thus probably not so great as to make the simplification selected here for lack of better data no longer acceptable.

Non-renewable primary energy is obtained from the difference between total primary energy and renewable sources.

Table 37: Target energy consumption, EU-28

\begin{tabular}{|l|ll|}
\hline Target energy consumption in EU-28 for 2020 & $\mathrm{PJ} / \mathrm{y}$ & Source \\
Target primary energy consumption & 61,713 & Directive 2012/27/EU \\
Target renewable energy consumption & 8,422 & Directive 2009/28/EC \\
Target non-renewable primary energy consump. & 53,291 & Line 1 minus line 2 \\
\hline
\end{tabular}

Applying the calculation specification, an eco-factor for consumption of "non-renewable energy sources" may be calculated (cf. Ahbe et al. 2014). Amounting to 8422 $\mathrm{PJ} / \mathrm{y}$ for 2020 , this constitutes $13.6 \%$ of what will then be the definitive target primary energy consumption of $61713 \mathrm{PJ} / \mathrm{y}$. In order to determine the eco-factor for "renewable energy consumption", the value of the eco-factor for consumption of "non-renewable primary energy" is reduced by this value of $13.6 \%$, resulting in a value of 0.01917 EP/MJeq. 
Table 38: EU-28 Eco Factors for energy consumption

\begin{tabular}{|l|l|}
\hline Reference year 2020 & Eco-factor in EP, EU-28/MJeq \\
Renewable energy consumption & 0.01917 \\
Non-renewable primary energy consumption & 0.02219 \\
\hline
\end{tabular}

First note: The time horizon for the primary energy consumption objectives is 2050 for the German data set approved by the UBA and 2020 for the remainder of Europe. No targets extending beyond that point have (yet) been defined at a European level. The target of reducing European greenhouse gas emissions by $80-95 \%$ by 2050 , does not allow any direct conclusions to be drawn regarding a reduction target for primary energy over the same period, since there are various sources of greenhouse gases and $\mathrm{CO}_{2}$ storage technology cannot be ruled out for this time horizon. While the EU is indeed responsible for defining objectives in the field of greenhouse gas emissions, energy policy is a matter for the individual countries, which explains the absence of more far-reaching, binding objectives in European energy policy.

Second note: The values listed above solely reflect the energy scarcity situation. When calculating the entire environmental impact caused by consuming non-renewable (in particular fossil) energy, it is additionally necessary to take account of the other environmental impacts such as pollutant emissions by applying the corresponding Eco Factors stated above (cf. Ahbe et al. 2014).

\subsection{Waste Generation}

\subsubsection{Non-Hazardous and Hazardous Waste}

In addition to the intended reduction in waste volumes, the European Commission is also planning to move towards resource cycle management, in which waste becomes a raw material again, so changing the meaning of the word "waste". The target is to utilise the greatest possible proportion of the generated waste streams by means of material recycling or energy recovery ${ }^{3}$.

Hazardous and non-hazardous waste is treated separately from a waste management standpoint and separate Eco Factors have accordingly been determined.

A distinction is drawn below between

non-hazardous waste and

hazardous waste.

3 cf. "Being wise with waste: the EU's approach to waste management", European Union 2010 
The volume of non-hazardous waste is determined by subtracting hazardous waste, which is considered separately, from the total volume of waste generated. Waste from mining, from the extraction of rock and ore and from the construction sector are also subtracted because they are largely mineral in nature and virtually unreactive.

With regard to the objectives for both non-hazardous and hazardous waste, it is assumed, as in the German Federal Environment Agency definition (cf. Ahbe et al. 2014), that the present day waste volumes described above should be considered more or less critical and should therefore not be allowed to rise further in future. In other words, the current flow and critical flow are identical and this applies to both classes of waste. In line with a written statement from the EEA in July 2014, this assessment is also applied at EU-28 level (EEA 2014c).

The following waste volume values are obtained:

Table 39: Waste generation, EU-28

\begin{tabular}{|l|ll|}
\hline Waste generation, EU-28 & Mt/y & Source \\
Non-hazardous waste, 2010 & 893.5 & Eurostat 2014c \\
Hazardous waste, 2010 & 94.46 & Eurostat 2014c \\
\hline
\end{tabular}

Table 40: Critical waste volume, EU-28

\begin{tabular}{|l|ll|}
\hline Critical waste volume, EU-28 & Mt/y & Source/details \\
Non-hazardous waste & 893.5 & EEA, July 2014 \\
Hazardous waste & 94.46 & EEA, July 2014 \\
\hline
\end{tabular}

Applying the calculation specification, the following Eco Factors for waste are obtained:

Table 41: Eco Factors for waste, EU-28

\begin{tabular}{|l|l|}
\hline & Eco-factor in EP, EU-28/g \\
Non-hazardous waste & 0.00112 \\
Hazardous waste & 0.01059 \\
\hline
\end{tabular}




\subsection{Derived Data Sets for Individual EU Countries}

\subsubsection{References to Calculation in the Datasheets}

\section{General}

ISO 3166 alpha-2 country codes are used.

The data sets have not been coordinated in this form with the authorities of the individual countries (cf. item 3.3.5).

Calculation of individual values

- The EU target is to reduce greenhouse gas emissions by $80-95 \%$ from their 1990 levels by 2050 . The $80 \%$ target has been selected for the individual countries too. Should it prove possible to "exceed" the target reduction, the target value can be further adjusted on subsequent review.

The Deltares report data originate from 2005-2010, those for E-PRTR from 2012.

The conversion factor for TOC into COD is 3, a value which was obtained from the E-PRTR database (European Environment Agency 2014a).

EPA-PAH16: the Deltares report lists only anthracene and fluoranthene. Since there is no suitable method with which total PAH16 emissions can be estimated on the basis of these two substances, the PAH16 values for the individual countries have been provisionally extrapolated pro rata to population from the German values.

Freshwater: the OECD stress limit of $20 \%$, indicating what is considered to be the critical flow, has been applied to all countries.

Specific features of the values for individual countries

Extrapolation of emissions to water for Malta and Croatia:

- Croatia: is not yet included in the Deltares report and as yet has no entries in the E-PRTR database. Values for emissions to water in Croatia are therefore calculated pro rata to population (2014) on the basis of determined total EU-27 emissions (excluding Malta) (source for population sizes: Eurostat).

- Malta: since the Deltares report lists too few values for Malta and the E-PRTR database likewise states no emission values for the pollutants (too few large industrial plants with an obligation to report), there is no reasonable basis for calculating emissions to water. Values were therefore extrapolated pro rata to population in the same way as for Croatia.

Waste in Estonia and Bulgaria: since the single value for hazardous waste in Estonia is very high, the EU average pro rata to population was used instead, thereby evening out the data on a plausibility basis. No further investigations were carried out into the causes of the data deviations. Since the value for mining waste in the statistics for Bulgaria is very high, a very low value for non-hazardous waste is ob- 
tained. The EU average pro rata to population for mining waste was therefore used instead. This, however, in turn resulted in a relatively high value for non-hazardous waste. No further investigations were carried out into the causes of the data deviations.

Renewable energy targets: on the basis of existing data, the calculated target value has already been exceeded in some countries (Czech Republic, Estonia, Croatia, Italy, Latvia, Lithuania, Hungary, Austria, Poland, Portugal, Romania, Slovakia, Finland and Sweden). This has been noted as a positive development and has not been further investigated.

Freshwater consumption in Cyprus: the relatively high value for the eco-factor is obtained because Cyprus' freshwater resources, with water consumption index of $63 \%$, are under severe stress.

Freshwater consumption in Croatia: the average values for Italy, Slovenia and Greece as neighbouring countries were applied pro rata to population to Croatia. The reference countries were selected to provide similar climatic conditions.

Freshwater consumption in the UK: since the source used only gives details for England and Wales, a value pro rata to population was extrapolated to the UK.

Open Access This chapter is licensed under the terms of the Creative Commons Attribution 4.0 International License (http://creativecommons.org/licenses/by/4.0/), which permits use, sharing, adaptation, distribution and reproduction in any medium or format, as long as you give appropriate credit to the original author(s) and the source, provide a link to the Creative Commons license and indicate if changes were made.

The images or other third party material in this chapter are included in the chapter's Creative Commons license, unless indicated otherwise in a credit line to the material. If material is not included in the chapter's Creative Commons license and your intended use is not permitted by statutory regulation or exceeds the permitted use, you will need to obtain permission directly from the copyright holder.

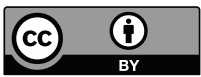

